\title{
Umfrage des Vereins der Leitenden Spitalärzte der Schweiz VLSS: Basismitgliedschaft in der FMH?
}

Hans-Ueli Wuersten, Präsident VLSS

Thomas Eichenberger, Geschäftsleiter VLSS

\section{Kaderärzte in der Schweiz}

Der Verein der Leitenden Spitalärzte der Schweiz VLSS umfasst die in der Schweiz an den Spitälern in leitender Funktion tätigen Kaderärzte (Chefärztinnen und Leitende Ärzte). Der Verein ist in kantonale Sektionen gegliedert, welche entsprechend der Anzahl der in der Sektion vertretenen Mitglieder Delegierte in das oberste Entscheidungsorgan (Delegiertenversammlung) entsenden.

Wir gehen davon aus, dass in der Schweiz mindestens 2500 und 3000 Ärztinnen und Ärzte in leitender Position tätig sind. Es handelt sich primär um an Spitälern mit Ausbildungsfunktion und Leistungsauftrag im Rahmen der Sozialversicherung tätige, angestellte Spitalärzte (meist öffentliche Spitäler). Angestellte Spitalärzte in leitender Funktion können neu auch Mitglied beim VLSS werden, wenn sie an Spitälern ohne Ausbildungs- und/oder Leistungsauftrag tätig sind (Privatspitäler).

Der VLSS vertritt gemäss Art. 20 der FMHStatuten die Anliegen der am Spital tätigen Chefärztinnen bzw. Chefärzte und Leitenden Ärztinnen bzw. Leitenden Ärzte gegenüber Bevölkerung, Behörden und anderen Institutionen. Der Verein VLSS umfasst momentan rund 1000 Mitglieder.

\section{Stellung innerhalb der FMH}

Der VLSS ist mit einem Sitz im Zentralvorstand der FMH vertreten. Innerhalb der Ärztekammer hat der VLSS lediglich die Funktion und das Gewicht einer Fachgesellschaft (2 Delegierte).

Diese im Verhältnis zu den obenerwähnten Aufgaben mangelhafte Vertretung im obersten Entscheidgremium der FMH hat den VLSS dazu bewogen, in der Ärztekammer in kurzer Abfolge zweimal den Antrag auf Anerkennung als Basisorganisation zu stellen. Dieser Antrag wurde, wenn auch beim zweiten Mal weniger deutlich, von der Ärztekammer abgewiesen.
Übriggeblieben ist der Auftrag der Ärztekammer, einen Zusammenschluss mit dem VSAO in einer Dachorganisation der Spitalärzte zu prüfen. Diese Arbeiten schreiten voran. An der nächsten Ärztekammer soll darüber Bericht erstattet werden.

\section{Weitere Entwicklung}

Damit bleibt aber das eigentliche Problem, die mangelhafte Vertretung des VLSS in der Ärztekammer, insbesondere aber die mangelhafte Identifizierung der Kaderärzte mit den kantonalen Ärztegesellschaften als Basisorganisation, in denen sie Mitglied sein müssen, bestehen.

Die kantonalen Ärztegesellschaften sind auf die konstruktiven Vorschläge des VLSS bisher nicht oder zu wenig eingegangen. So wäre es unter anderem denkbar, den VLSS als Basisorganisation zu verankern, gleichzeitig aber die obligatorische Mitgliedschaft in den kantonalen Ärztegesellschaften aufrechtzuerhalten. Die Mitgliederbeiträge könnten entsprechend gesplittet werden.

Unter den gegebenen Umständen ist zu befürchten, dass die Kaderärzte zumindest in einigen Kantonen aus der kantonalen Ärztegesellschaft austreten könnten.

\section{Umfrage}

Umfrage betreffend Basisorganisation VLSS Der VLSS hat deshalb im Sommer 2003 eine Umfrage bei sämtlichen in der Schweiz tätigen Kaderärztinnen und Kaderärzten durchgeführt.

Der Rücklauf war nicht berauschend, aber doch befriedigend, haben doch bei 2750 versandten Fragebogen deren 804 Teilnehmerinnen und Teilnehmer den Fragebogen ausgefüllt zurückgeschickt. Der Rücklauf betrug somit gut $29 \%$ oder fast $1 / 3$. Fast die Hälfte der Teilnehmenden sind Nichtmitglieder des VLSS (368), etwas mehr als die Hälfte sind Mitglieder des VLSS (436). 
Abbildung 1

Umfrage betreffend Basisorganisation des VLSS bei der FMH.

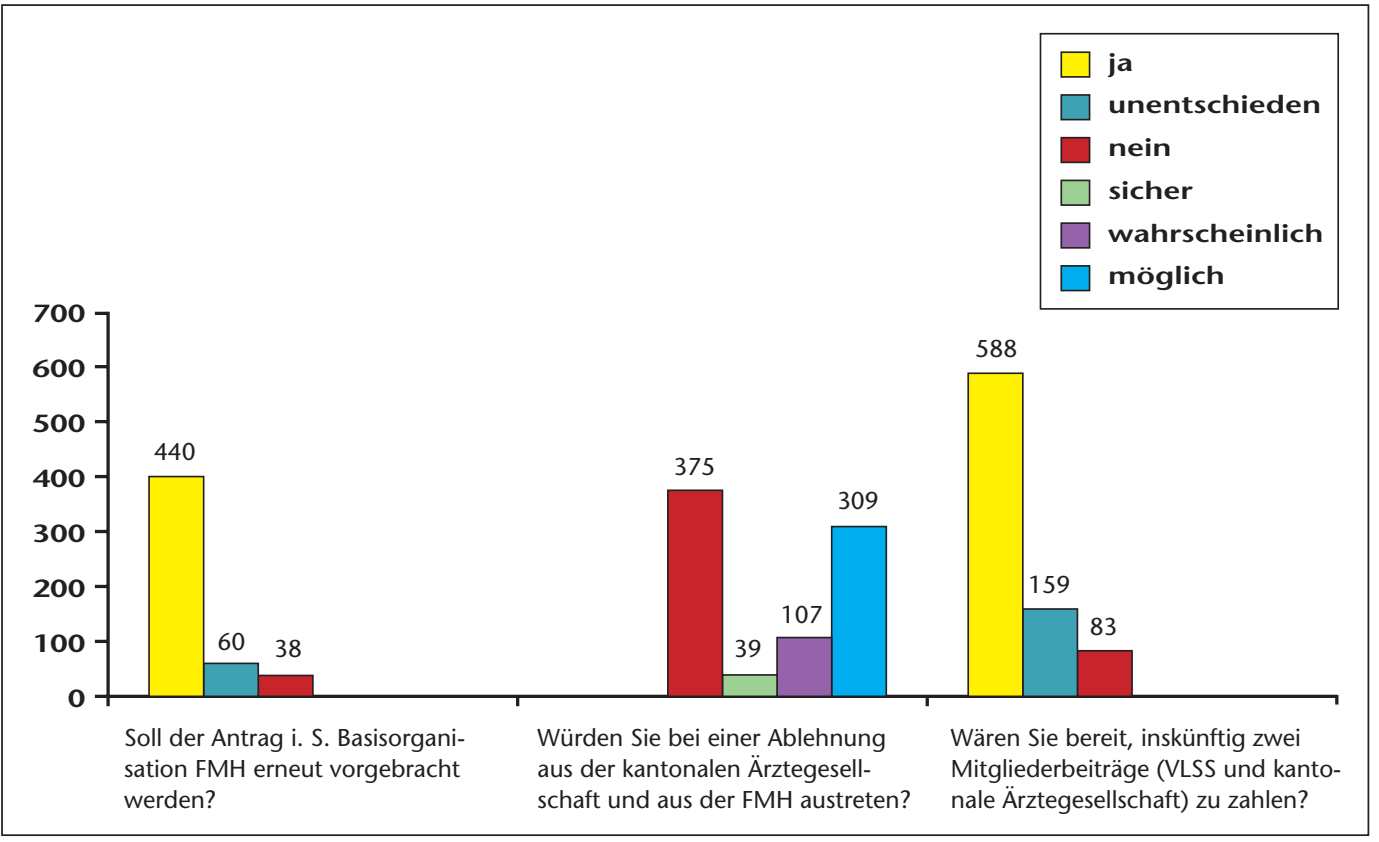

Abbildung 2 Umfrage betreffend Aktivitäten und Dienstleistungen des VLSS.

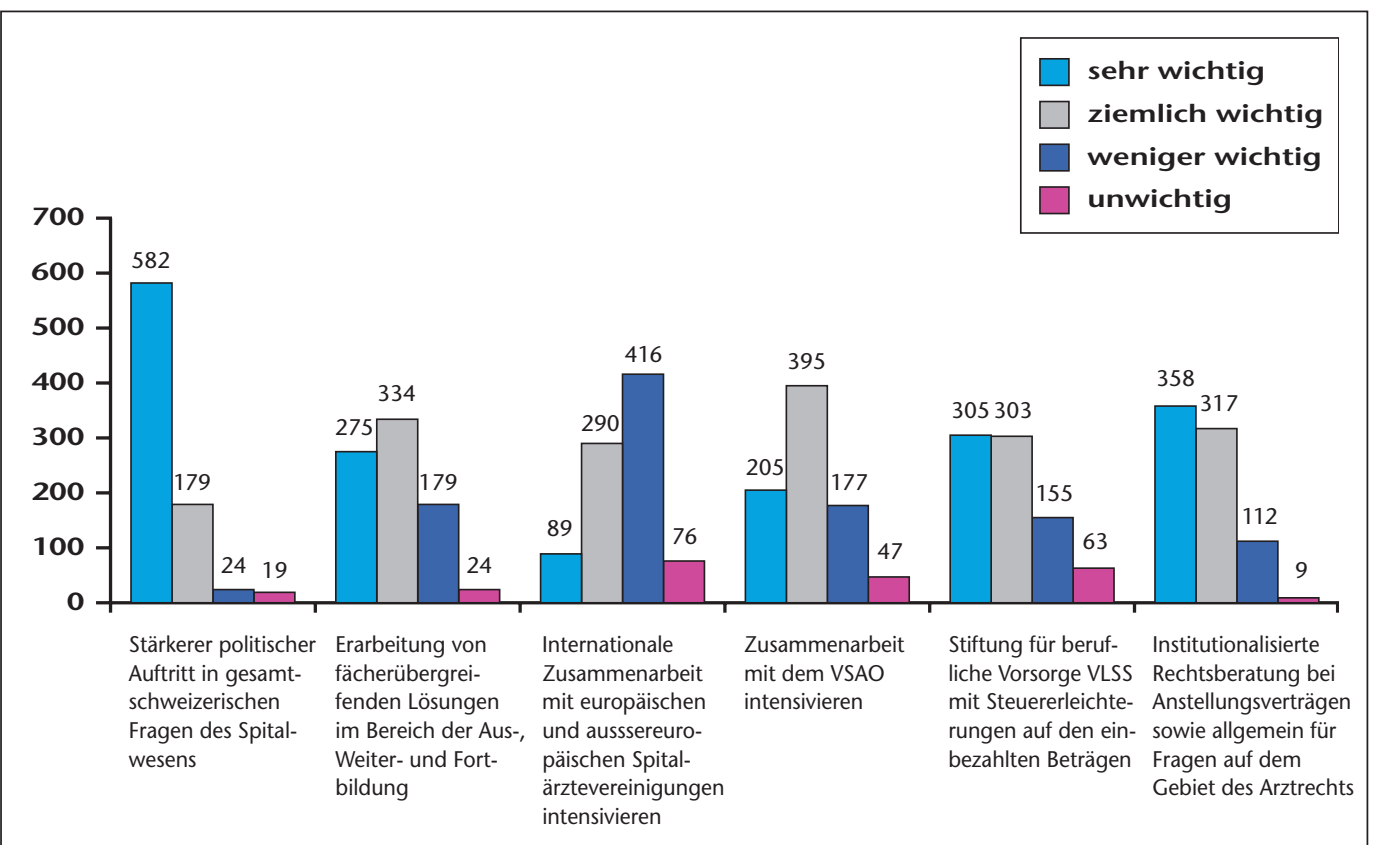

Die Beantwortung der gestellten Fragen können Sie der Abbildung 1 entnehmen.

Demzufolge spricht sich die Basis der Kaderärzte grossmehrheitlich dafür aus,

- den Antrag auf Anerkennung in der Ärztekammer als Basisorganisation erneut zu stellen und

- zwei Mitgliederbeiträge zu zahlen, einen reduzierten Mitgliederbeitrag bei der kantonalen Ärztegesellschaft und den Mitgliederbeitrag VLSS (momentan Fr. 150.-), sofern die Aufnahme des VLSS als Basisorganisation durch die Ärztekammer nur unter dieser Voraussetzung (gleichzeitige Mitgliedschaft in der kantonalen Gesellschaft obligatorisch) möglich ist.

Die Frage, ob sie bei erneuter Ablehnung des Antrages um Aufnahme des VLSS als Basisorganisation durch die Ärztekammer aus der FMH austreten würden, haben die Kaderärztinnen und Kaderärzte unterschiedlich beantwortet.

- 375 der Befragten (etwa 46,6\%) würden nicht aus der FMH austreten; 
- nur 39 (etwa 4,9\%) würden dagegen sicher aus der FMH austreten;

- immerhin 309 der Befragten (etwa 38,4\%) würden wahrscheinlich aus der FMH austreten und

- 107 (etwa 13,3\%) sind noch unentschieden.

Bemerkung zu den Prozentzahlen: Mehrfachnennungen waren nicht erwünscht, wurden aber dennoch ausgewertet, so dass bei 804 Teilnehmern insgesamt 830 Nennungen berücksichtigt wurden.

\section{Umfrage betreffend Aktivitäten und Dienstleistungen VLSS}

Wir haben die Gelegenheit ergriffen, die Basis in einem zweiten Teil darüber zu befragen, welche Aktivitäten und Dienstleistungen die Kaderärztinnen und Kaderärzte als am wichtigsten einstufen. Das Ergebnis können Sie der Abbildung 2 entnehmen.

Als sehr wichtig wurden der stärkere politische Auftritt in gesamtschweizerischen Fragen des Spitalwesens (582 Nennungen oder 72,3\%) und das Angebot einer institutionalisierten Rechtsberatung (358 Nennungen oder etwa $47 \%)$ eingestuft.

Als ziemlich wichtig werden bezeichnet

- die Zusammenarbeit mit dem VSAO (395);

- die Erarbeitung von fächerübergreifenden Lösungen im Bereich der Aus-, Weiter- und Fortbildung (334) und

- die Stiftung für berufliche Vorsorge VLSS, welche Steuererleichterungen auf den einbezahlten Beiträgen ermöglicht (303).

Die internationale Zusammenarbeit wurde mehrheitlich als weniger wichtig beurteilt (416 Nennungen).

\section{Vorläufige Schlussfolgerungen}

Es kann nicht darum gehen, an dieser Stelle mit einem Kampfartikel aufzutreten. Vielmehr soll die Umfrage an der Basis eine gewisse Ausrichtung unserer Politik der nächsten Jahre definieren. So unzufrieden mit der Verbindung der Schweizer Ärztinnen und Ärzte FMH sind offenbar unsere Mitglieder nicht, als dass sie in grossem Stil die FMH verlassen würden. Ein möglicherweise markant anderes Bild dürfte sich allenfalls zumindest da und dort ergeben, wenn es um die Mitgliedschaft in kantonalen Ärztegesellschaften geht.
Es ist somit klar, dass auch die Politik des VLSS in Richtung Zusammenarbeit und Konsens gehen muss, damit ein möglichst kraftvolles Auftreten der Ärzteschaft als «Frontverantwortliche» möglich ist. Dies setzt allerdings voraus, dass in zukünftigen strukturpolitischen Fragen der Verbindung das tatsächliche Gewicht der Chef- und Leitenden Ärzte und Ärztinnen, welches sich aus der Gesamtressourcenverantwortung im Gesundheitswesen leicht ablesen lässt, auch gebührend in den Strukturen der FMH berücksichtigt wird. Auch wenn die Statistik eine gefährliche Sache sein kann, lässt sich doch unschwer aus den Zahlen dieser Studie zumindest ein «Unwohlsein» der Leitenden Spitalärzte innerhalb der bisherigen Strukturen feststellen.

Dass ein näheres Zusammengehen mit einer weiteren Gruppe der Spitalärzte, nämlich der Gruppe der Assistenz- und Oberärzte, in Zukunft ein Muss ist, wird von unseren Mitgliedern offenbar ziemlich gut erkannt. Die schärferen Randbedingungen, die durch das Arbeitsgesetz für die Assistenz- und Oberärzte Einzug halten, bedingen eine wesentlich klarere Strukturierung der Arbeits- und Bildungsabläufe innerhalb eines Spitals, damit trotz grossem Arbeitsaufwand im Dienstleistungssektor die Weiterbildung zu kompetenten Fachärztinnen und Fachärzten durch neue Randbedingungen nicht kompromittiert wird.

Es sei an dieser Stelle gleich festgehalten, dass es das Bemühen der Spitalärzte insgesamt - seien dies Chefärzte, Leitende Ärzte, Oberärzte oder Assistenzärztinnen und -ärzte - sein muss, möglichst rationelle und optimierte Arbeitsabläufe anzustreben; andererseits gilt es auch klar festzuhalten, dass sich die politische Instanz mit dem zunehmend eher unqualifizierten Hinweis auf organisatorische Massnahmen nicht so einfach aus der Verantwortung stehlen kann. Qualitativ hochstehende Ärztinnen und Ärzte im Gesundheitswesen ist eine Kernverantwortung, auch der staatlichen Instanz, und damit sind zusammen mit dem freudvollen Ja zum Arbeitsgesetz auch die allenfalls notwendigen Ressourcen zu sprechen.

Dass ein zunehmendes Bedürfnis nach sozialer Absicherung in der Altersvorsorge ein Anliegen unserer Mitglieder ist, dürfte ganz wesentlich mit den immer straffer werdenden finanziellen Bedingungen unserer Mitglieder zu tun haben; ein Anliegen, das mehr als berechtigt ist und dem wir in Zukunft ein sehr scharfes Augenmerk widmen werden. 